Journal of Sustainable Agricultural Sciences
http://jsas.journals.ekb.eg/

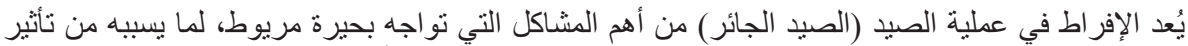

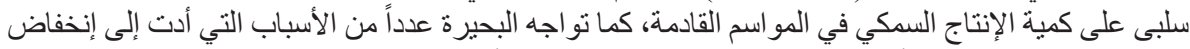

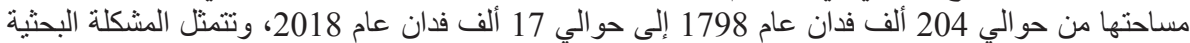

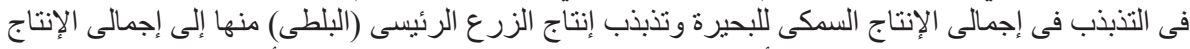

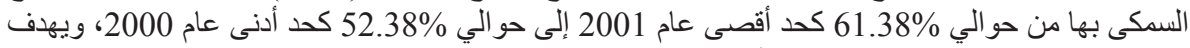

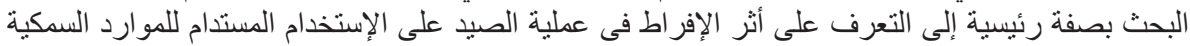

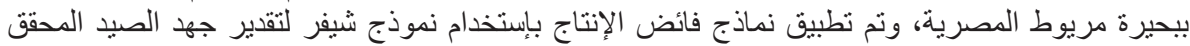

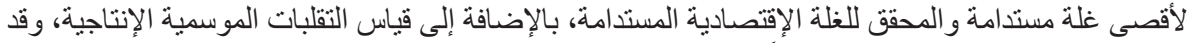

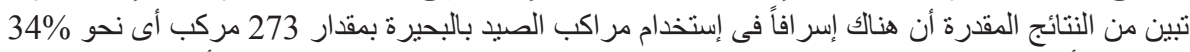

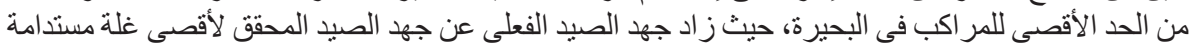

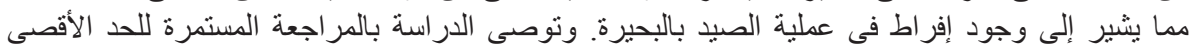

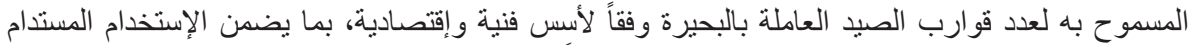

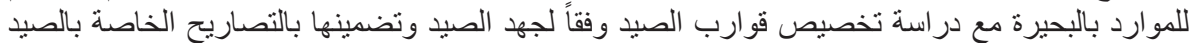
وذللك بالتعاون بين إدارة البحيرة و التعاونيات السمكية وشيو خ الصيادين بالبحيرة.

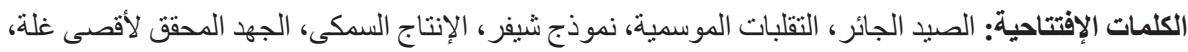
بحيرة مريوط، الموارد السمكية.

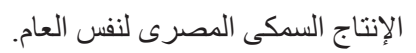

ويعد الإفر اطفى عملية الصيد (الصيد الجائر) من أهم المشاكل

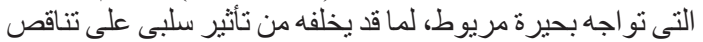

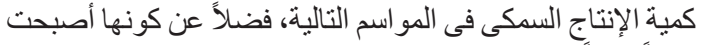

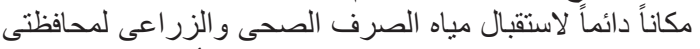

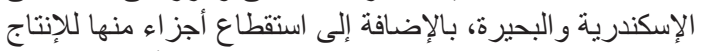

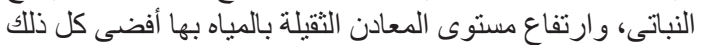
إلى تذبذب إنتاجها من عام لآخر.

المشكلة البحثية

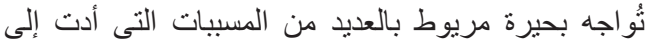

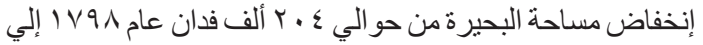

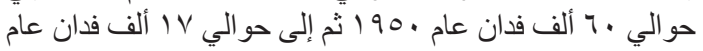

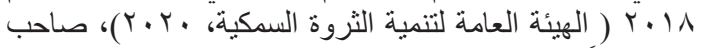

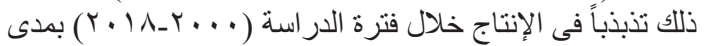

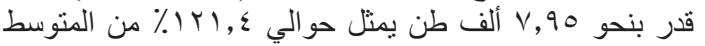

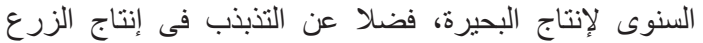

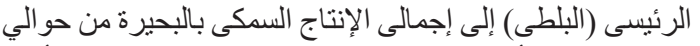

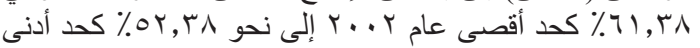

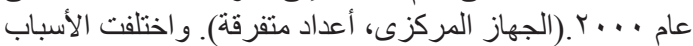

المقدمة

تُعد الممارسات غير المستدامة لصيد الأسماك ومنها الإفراط في بلي

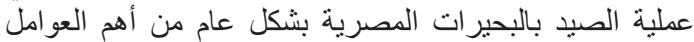

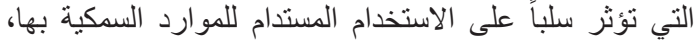

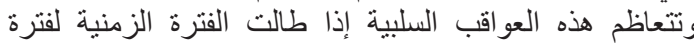

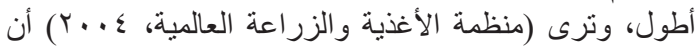

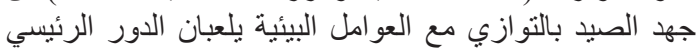

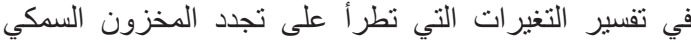

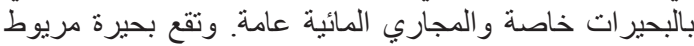

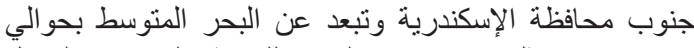

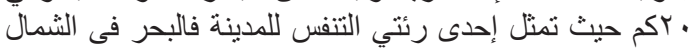

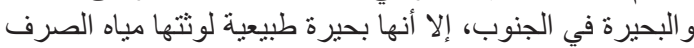

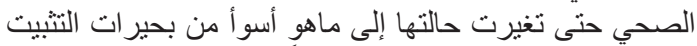

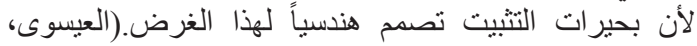

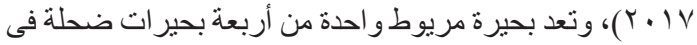

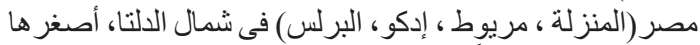

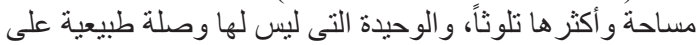

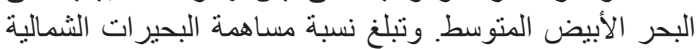

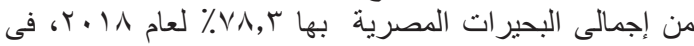

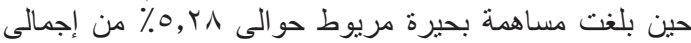

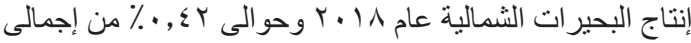




$$
\mathrm{f}_{\mathrm{i}}=-0.5 \mathrm{a} / \mathrm{b}
$$

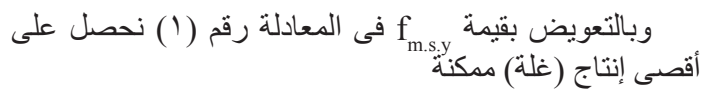

$\mathrm{y}_{\mathrm{i}}=-0.25 \mathrm{a}^{2} / \mathrm{b}$

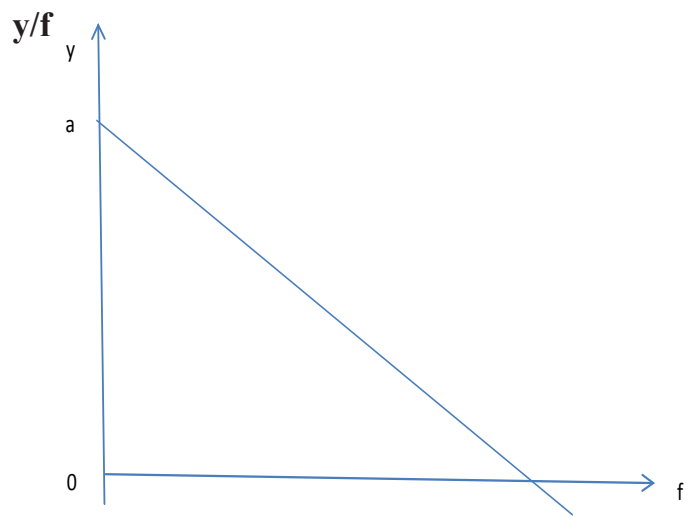

$-\mathbf{a} / \mathbf{b}$

Source: Sparre and Venema (1998).

$$
\text { شكل رقم (1) - نموذج شيفر }
$$

يفترض نموذج شيفر وجود علاقة عكسية بين انتاجية وجها

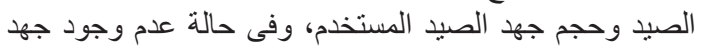

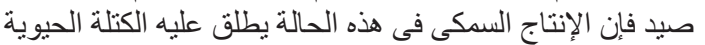

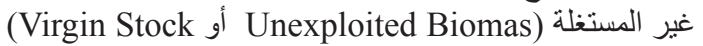
بالإطiomas، كما هو موضح بالنقطة (a ) بالثكل رقم (1).

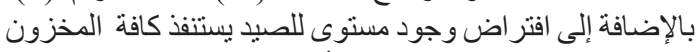

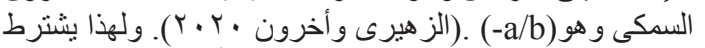

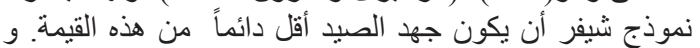

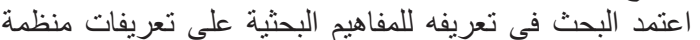

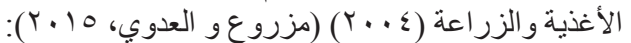

جهد الصيد:Catch Effort يقصد به ما يخصص من الوقت و القوة لصبد الأسماك، ووفقاً

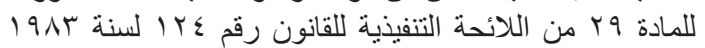

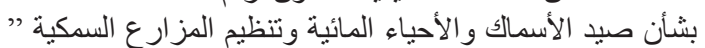

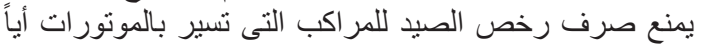

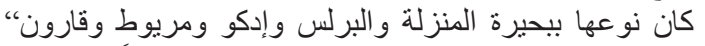

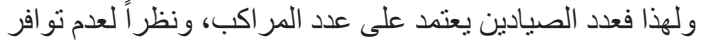

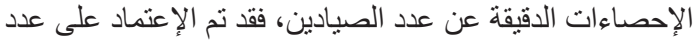
مر اكب الصيد فى السنة كمقياس لجهد الصيد.

Catch pre Unite Effort (CPUE) المصيد لكل وحدة جها يتم الحصول عليه من خلال قسمة طاقة الصيد السنوية على لك

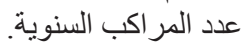

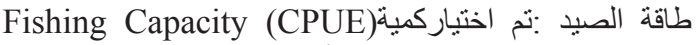
المصيد السنوية لبحيرة مريوط الأسماك بالطن للتعبير عن طاقة من هو حالة تحدث عندما تتعدى طاقة الصيد الصيد الصيد.

إلصيد الجائر ( الدفرط) الفعلية طاقة Overexploitation آلصيد القصوى المقدرة بنموذج شيفر.
حول هذا الإنخفاض، فأرجعته المصادر الرسمية إلى طرق الصيد

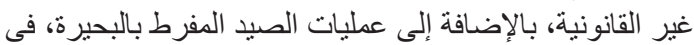

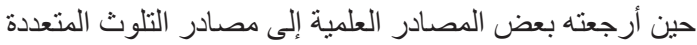

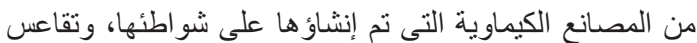

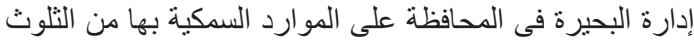

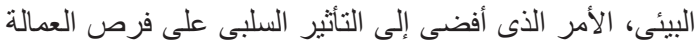

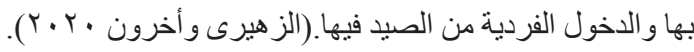

الأهداف البحثية

$$
\begin{aligned}
& \text { يرهذ البحث بصفة رئيسية إلى التعرف على أثر الإفراط }
\end{aligned}
$$

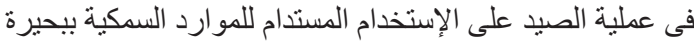

$$
\begin{aligned}
& \text { مريوط المصرية، مما تطلب دراسة الأهداف الفر عية التالية: } \\
& \text { - - تطور مصادر الإنتاج السمكى فى مصر. }
\end{aligned}
$$

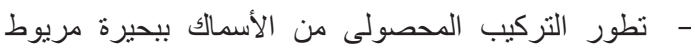

$$
\begin{aligned}
& \text { و التغير ات التى طر أت عليه خلال فترة الدر اسة. } \\
& \text { - تقدير جها الصيد المحقق لأقصى غلة مستدامة، والمحقق } \\
& \text { للغلة الإقتصادية المستدامة. } \\
& \text { - ق قياس التقلبات الموسمية الإنتاجية ببحيرة مريوط. }
\end{aligned}
$$

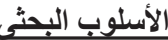

تحقيقاً لأهداف الدراسة، فقد تم الإعتماد على أساليب التحليل

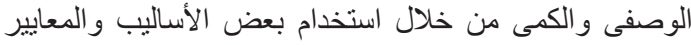

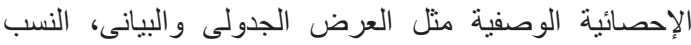

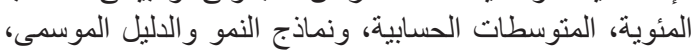

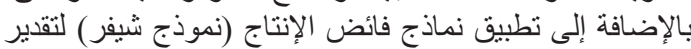
المستوى الأمثل لجهد الصيد، وتقدير جهد الصيد اللازئ لإن لتحقيق كل

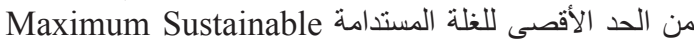
Economic)، والغلة الإقتصادية المستداملة:Y.S.Y)Yield جئو.S.Y) Sustainable Yield

$$
\text { لجهد الصيد للوقوف على حالة الصيد بالبحيرة. }
$$

وقد إقترح شيفر Schaefer (1954) نموذج خطى للعلاقة بين متوسط الإنتاج السمكى لوحدة الجهد ووحدة الجها ذاتها فى لـى

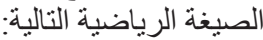

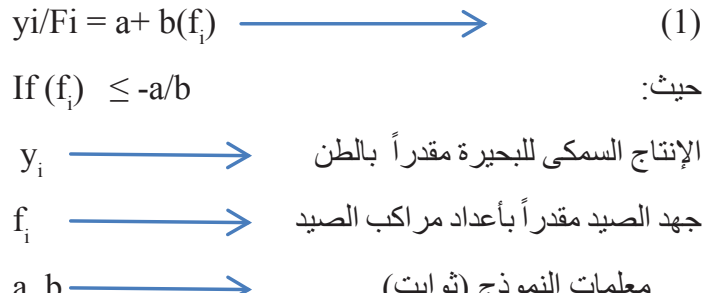

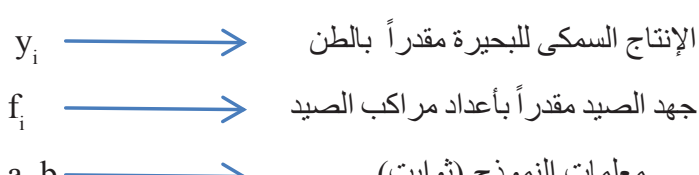

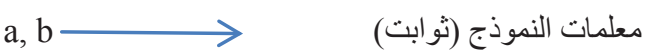

$$
\text { ويمكن تحويل المعادلة رقم ( ) إلى: }
$$

$\mathrm{Yi}=\mathrm{af}_{\mathrm{i}}+\mathrm{b}\left(\mathrm{f}_{\mathrm{i}}\right)^{2} \longrightarrow$

وبإجراء التفاضل الأول لهذه الدالة السابقة بالنسبة لجهـ الصيد للحصول على معادلة الناتج الحدى وبمساو اتها بالصفر نحصل على جها الصيد المحقق لأقصى غلة مستدامة (f). (f.y.

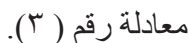




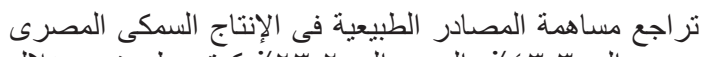

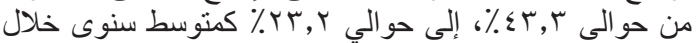

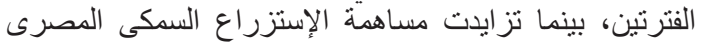

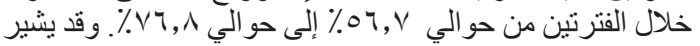

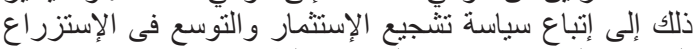
السكى على حساب تنمية المصادر الطبيعية.

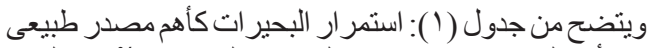

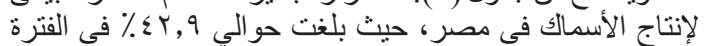

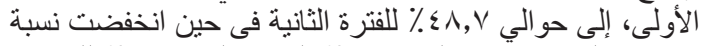

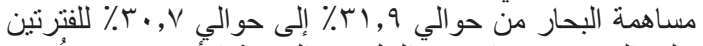

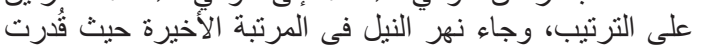

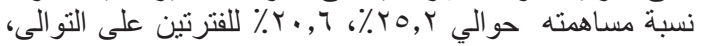

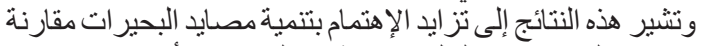

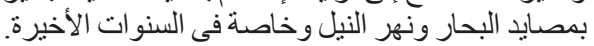

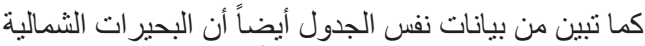

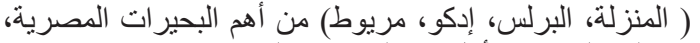

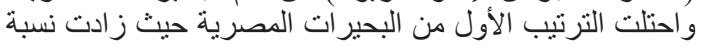

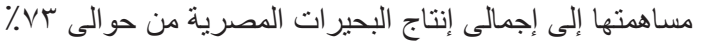

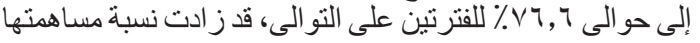

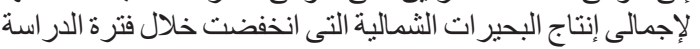

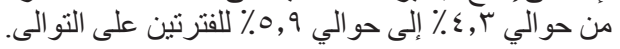

مصادر البيانات

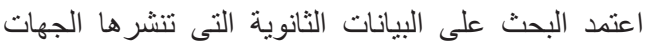

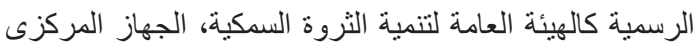

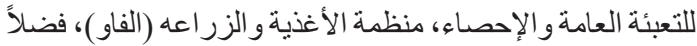

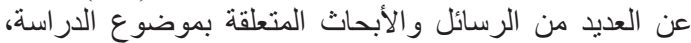

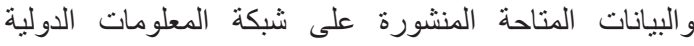

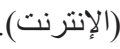

النتائج البحثية ومناقثتنها مصادر الإنتاج السمكى المصرى:

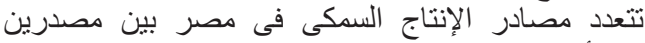

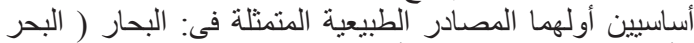

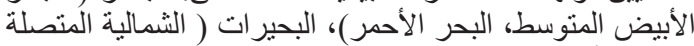
بالبحر الأبيض المتوسط، الداخلية، نهر النيل)، البحرات

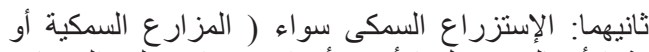

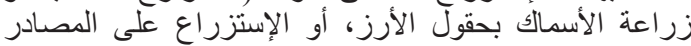

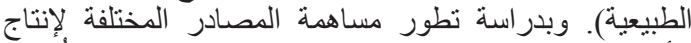

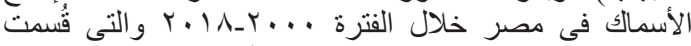

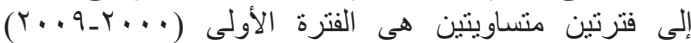

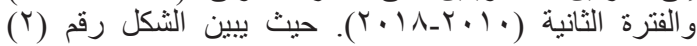

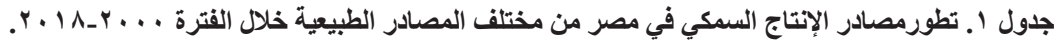

\begin{tabular}{|c|c|c|c|c|c|c|c|c|c|c|}
\hline \multicolumn{4}{|c|}{ البحيرات الثمالية } & \multicolumn{3}{|c|}{ البحيرات } & \multicolumn{3}{|c|}{ المصادر الطبيعية } & \multirow{3}{*}{ الفترة الزمنية } \\
\hline \multirow{2}{*}{ مريوط } & \multirow[b]{2}{*}{ أدكو } & \multirow[b]{2}{*}{ البرلس } & \multirow{2}{*}{ المنزلة } & \multicolumn{2}{|c|}{ من البحيرات } & \multirow{2}{*}{$\frac{\text { البحير ات }}{\text { الشمالية }}$} & \multicolumn{2}{|c|}{ من المصادر الطبيعية } & \multirow{2}{*}{ البحار } & \\
\hline & & & & المنخفضية المنات & الألخير ات & & نهر النيل & البحيرات & & \\
\hline$\varepsilon, r$ & $\mathrm{v}, 1$ & $\{0,1$ & $\varepsilon r, 0$ & r,r & $r \varepsilon, V$ & $v r$ & ro, r & $\varepsilon r, q$ & $r 1,9$ & $r \ldots q-r \ldots$ \\
\hline 0,9 & $\varepsilon, v$ & $\leq 0$ & $\varepsilon \varepsilon, \varepsilon$ & $r, r$ & $r, 1$ & 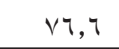 & $r \cdot, r$ & $\varepsilon \wedge, \vee$ & $r \cdot, v$ & $r \cdot \mid \Lambda_{-} r+1$. \\
\hline
\end{tabular}

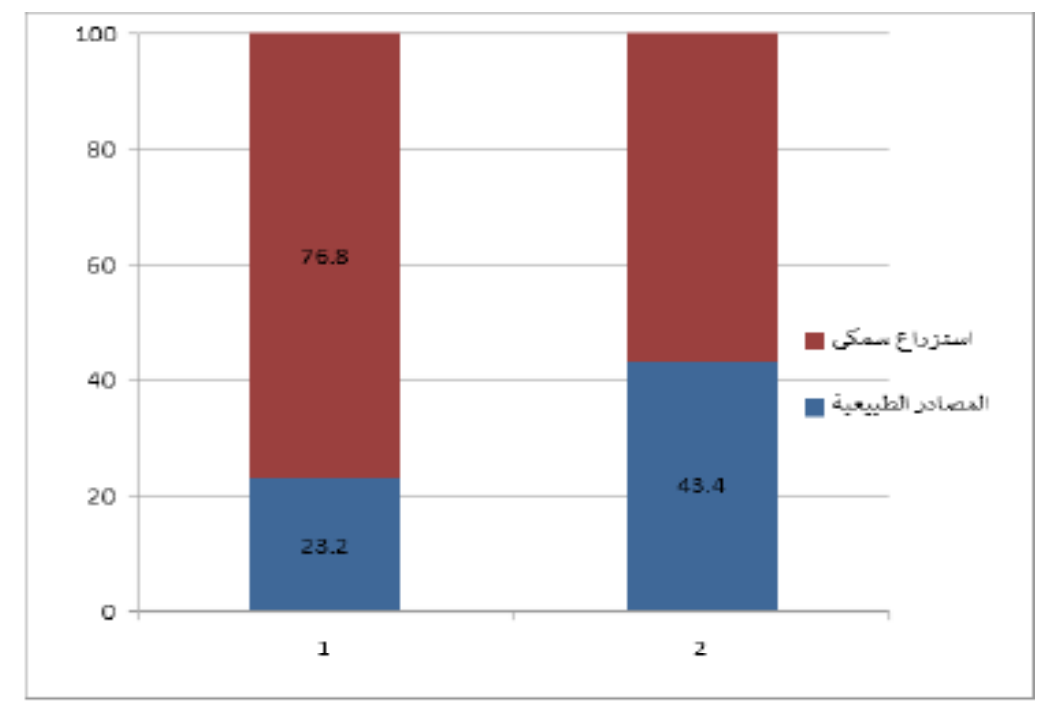

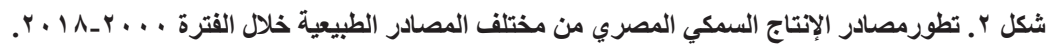

المصدر : بيانات جدول رقم (1) - (1).

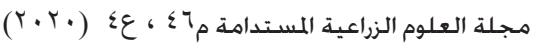




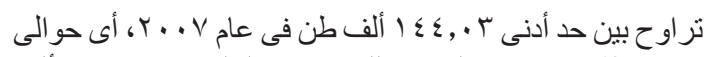

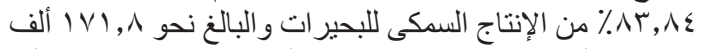

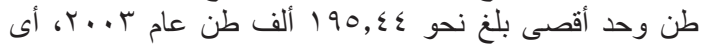

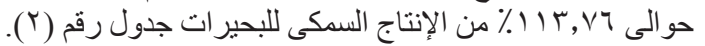

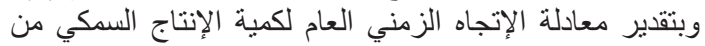
البحير ات تبين عدم معنوية هذا المتغير.

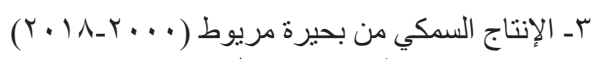

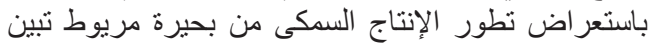

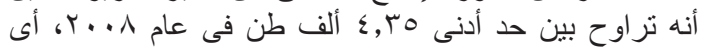

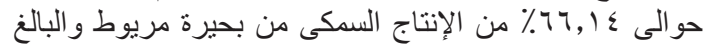

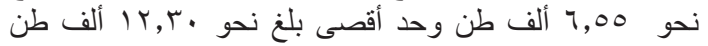

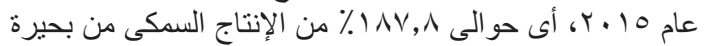

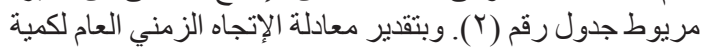

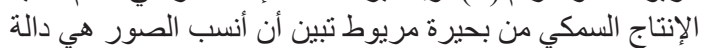

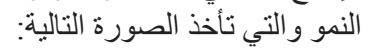

$\ln \hat{Y} \mathrm{i}=1.5+0.034 \mathrm{Ti}$

$$
\begin{array}{ll}
(15.52)^{* *} & (3.99)^{* *} \\
\mathrm{R}^{2}=0.485 & \mathrm{~F}=15.99 * *
\end{array}
$$

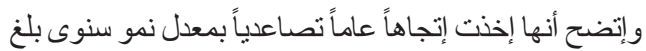

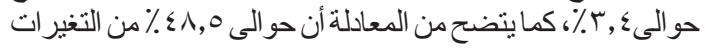

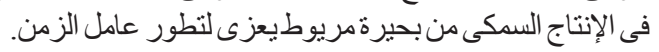

وقد تشير هذه النتائج إلى سوء إدارة بحيرة مريوط في الفترة

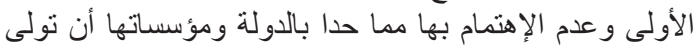

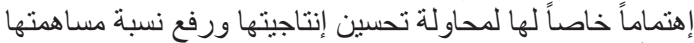

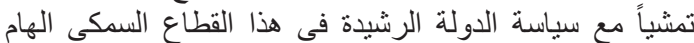
وخاصة في السنو ات الأخيرة.

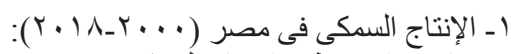

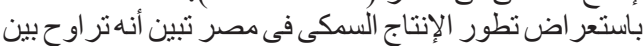

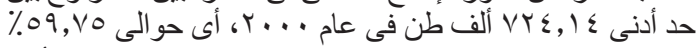

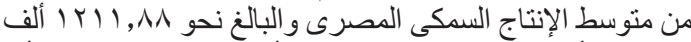

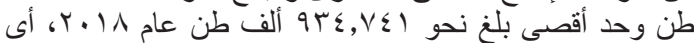

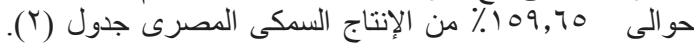

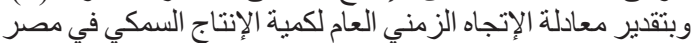
تبين أن أنسب الصور هي دالتة النمو والتي تأخذ الصواج الصورة التالية: $\ln \hat{\mathrm{Y}} \mathrm{i}=6.52+0.054 \mathrm{~T}$

$$
(369.9)^{* *} \quad(34.99)^{* *}
$$

$\mathrm{R}^{2}=0.986 \mathrm{~F}=1224.5^{* *}$ ** معنوى عند مستوى $1 *$

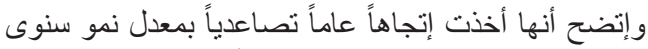

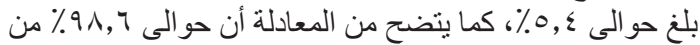

\begin{tabular}{|c|c|c|c|c|c|}
\hline \multicolumn{3}{|c|}{ كمية الإنتاج السمكى من بحيرة مريوط } & \multirow{2}{*}{ الإنتاج السمكى من البحيرات } & \multirow{2}{*}{ الإنتاج السمكى فى } & \multirow{2}{*}{ 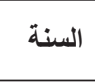 } \\
\hline إنتاج البحيرات٪ & إنتاج مصر \% & الإنتاج السمكى من مريوط & & & \\
\hline$r, 1 \Lambda$ & $\cdot, \wedge \wedge$ & $1, r \wedge$ & IVr,lo & 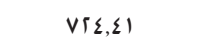 & $\uparrow \ldots$ \\
\hline$r, r \varepsilon$ & $\cdot \wedge$. & 1,5 & $1 \wedge 0, \Delta 9$ & VWI,OS & $\uparrow \cdots 1$ \\
\hline$r, \cdot \Lambda$ & $\cdot, 11$ & $\Delta, r$ & $\operatorname{IV} r, \varepsilon$ & $\Lambda \cdot 1, \sum \vee$ & $r \ldots r$ \\
\hline$r, \Sigma 9$ & $\cdot, \Delta$ & $\varepsilon, \wedge \uparrow$ & $190, \varepsilon \varepsilon$ & $\wedge \vee \Delta, 99$ & $r . . r$ \\
\hline$\ulcorner, \wedge \varepsilon$ & $\cdot, \Delta \Lambda$ & $0, \cdot r$ & $\operatorname{lvV}, \cdot 9$ & $\wedge 10, \cdot r$ & $r \cdots \varepsilon$ \\
\hline$r, r \varepsilon$ & $\cdot, 09$ & 0,59 & $10 \Lambda, 59$ & $\wedge \wedge 9, r$. & $r \cdots o$ \\
\hline$r, \leq \varepsilon$ & $\cdot, \Delta \varepsilon$ & 0,51 & $101, r 1$ & $9 V \cdot, 95$ & $\uparrow \cdots r$ \\
\hline$r, \cdot 1$ & $\cdot, \Sigma \varepsilon$ & $\varepsilon, \varepsilon 1$ & $1 \varepsilon \varepsilon, \cdot r$ & $1 \cdot \cdot \wedge, \cdot 1$ & $r \cdots v$ \\
\hline$\ulcorner, \mathrm{V} r$ & $\cdot, \Sigma)$ & $\varepsilon$, ro & $\perp \Delta \vee, \Lambda \Lambda$ & $1 \cdot \pi v, \pi$ & $r \cdots \wedge$ \\
\hline$r, r$. & $\cdot, 01$ & $0,0 \mathrm{r}$ & IVI, Is & $1.95, \wedge 9$ & $r \cdots q$ \\
\hline$r, r$. & $\cdot, \sum 0$ & 0,95 & $1 V 9,19$ & $1 r \cdot \varepsilon, v q$ & r.1. \\
\hline r.r. & $\cdot, \varepsilon$. & $0, \Sigma \mu$ & ع & vוזוזוז & {$[.11$} \\
\hline$\varepsilon, \Gamma \wedge$ & $\cdot, \Delta \varepsilon$ & $v, \varepsilon r$ & IVr, & $|r v|, 9 \Lambda$ & r.1r \\
\hline$\varepsilon, 19$ & ror & $v, 1 \varepsilon$ & $\mid \wedge \Gamma, \Delta \Gamma$ & $1 \varepsilon \Delta \varepsilon, \varepsilon$. & r.1r \\
\hline$\varepsilon, r V$ & $\cdot, 0$. & $v, \Sigma 1$ & IV,$q$. & $|\leq \wedge|, \wedge \Lambda$ & $r .1 \varepsilon$ \\
\hline$v, 1 \Lambda$ & $\cdot, \wedge 1$ & $15, r$. & $|v|, r$. & $101 \Lambda, 9 \varepsilon$ & 1.10 \\
\hline $0, \Sigma 1$ & $\cdot, 0$. & $\wedge, 01$ & $|\Delta \Lambda, r|$ & IV. $1, \mathrm{VV}$ & 5.11 \\
\hline$\varepsilon, 9 \mathrm{~V}$ & $\cdot, \Delta \cdot$ & 9,17 & $\mid \wedge r, \Sigma 1$ & $\mid \wedge[\Gamma, \wedge \mid$ & r.IV \\
\hline$\varepsilon, 1 \varepsilon$ & $\cdot, \sum \Gamma$ & $\Lambda, \cdot 1$ & $19 \varepsilon, \wedge \Delta$ & $19 \pi \varepsilon, V \varepsilon$ & $r .11$ \\
\hline$r, \wedge 1$ & $\cdot, 01$ & 1,00 & $|v|, \wedge \mid$ & $\|[\|, \wedge \Lambda$ & المتوسط \\
\hline
\end{tabular}
التغير ات فى الإنتاج السمكى المصرى يح يعزى لتطور عامل الزمن.

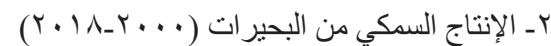

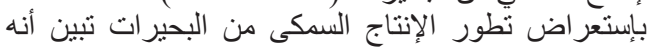

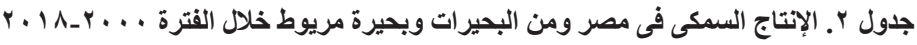




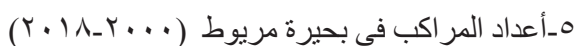

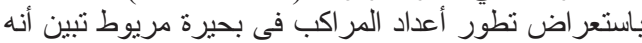

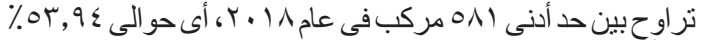

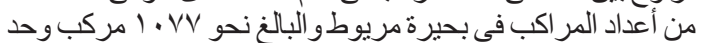

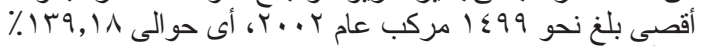

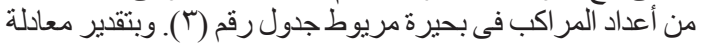

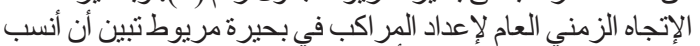
الصور هي دألّة النمو و التني تأخذ الصورة التباد التالية:

$\ln \hat{\mathrm{Y}} \mathrm{i}=7.3-0.034 \mathrm{Ti}$

(111.68) (-5.98)

$\mathrm{R}^{2}=0.678 \quad \mathrm{~F}=35.75 * *$

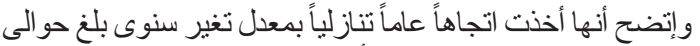

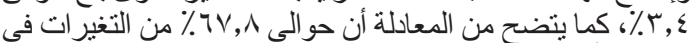

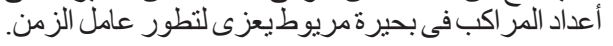

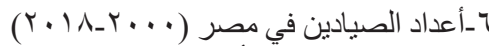

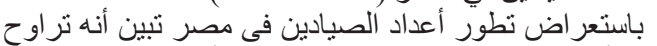

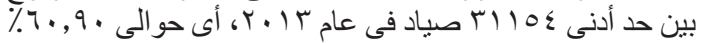

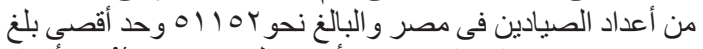

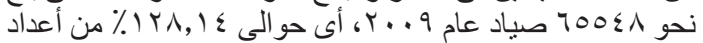

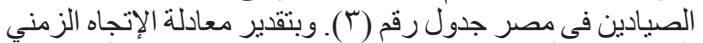

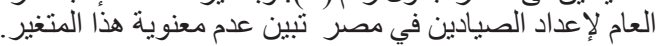

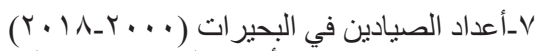

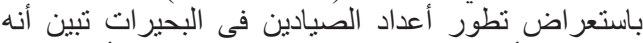

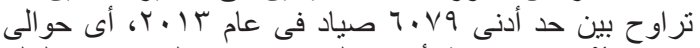

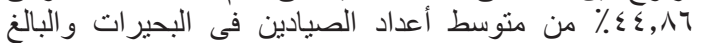

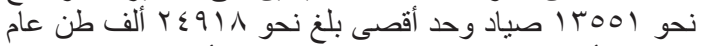

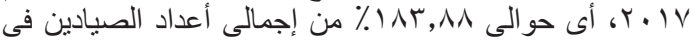

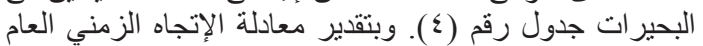
لإعداد الصيادين في البحير ات تبين عدم معنوية هذا المتغير.

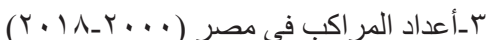

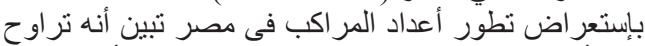

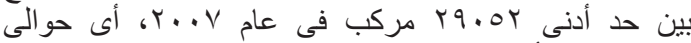

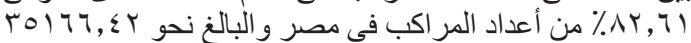

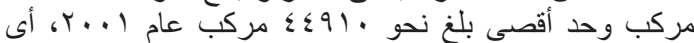

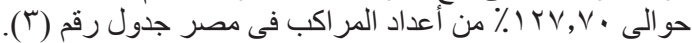

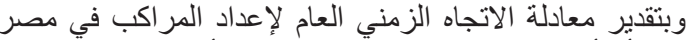

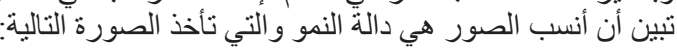
$\ln \hat{\mathrm{Y}} \mathrm{i}=10.64-0.019 \mathrm{Ti}$

$\left(179.21^{* *}\right) \quad(3.63)^{* *}$

$\mathrm{R}^{2}=0.436 \quad \mathrm{~F}=13.15 * *$

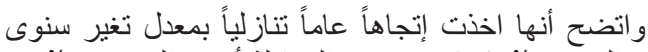

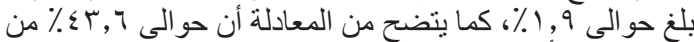

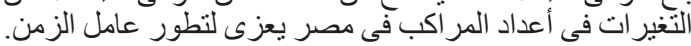

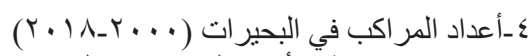

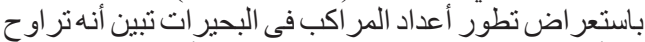

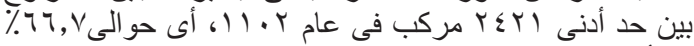

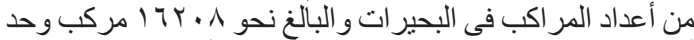

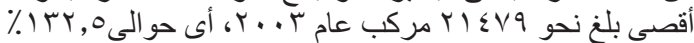

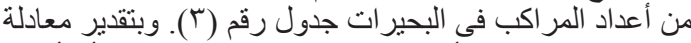

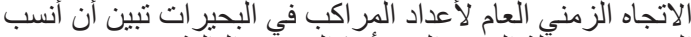
الصور هي دآلة النمو والتي تأخذ الصورئ الصورة النتالية:

$\ln \hat{\mathrm{Y}} \mathrm{i}=9.96-0.028 \mathrm{Ti}$

(178.36)

$\mathrm{R}^{2}=0.659 \quad \mathrm{~F}=32.9 * *$

و إتضح أنها أخذت إتجاهاً عاماً تنازلياً بمعدل تغير سنوى

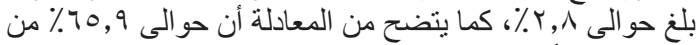

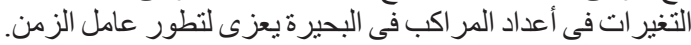

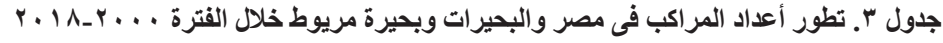

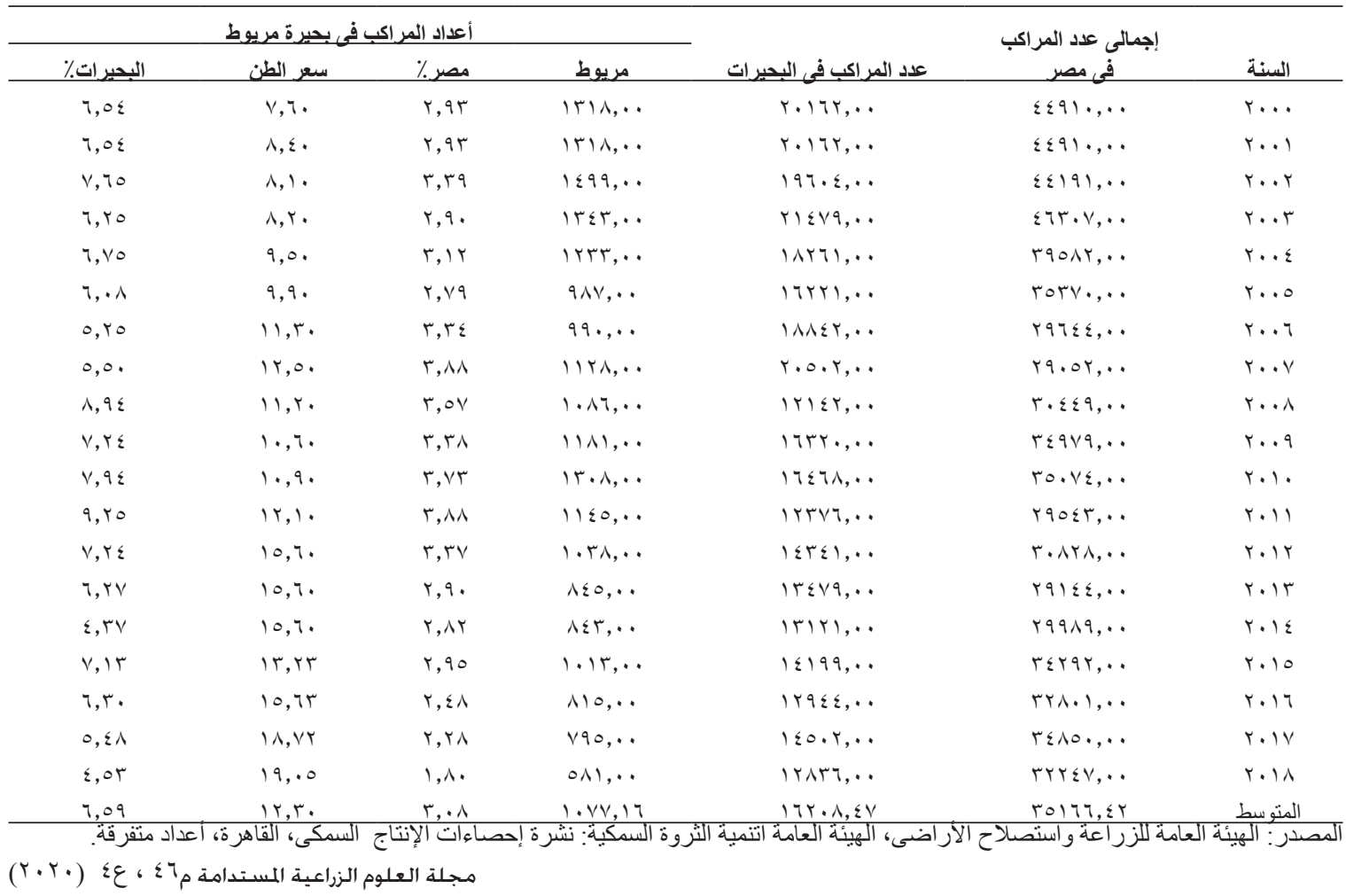




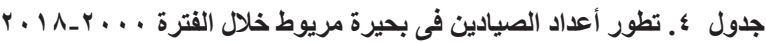

\begin{tabular}{|c|c|c|c|c|c|c|}
\hline \multicolumn{3}{|c|}{ أعداد الصيادين فى بحيرة مريوط } & \multirow{2}{*}{ المساحة } & \multirow{2}{*}{ إجمالى عدد صيادى } & \multirow{2}{*}{ إجمالى عدد صيادى } & \multirow{2}{*}{ ال السنة } \\
\hline البحيرات \% & مصر \% & مريوط & & & & \\
\hline$\cdot, \S 1$ & $\cdot, 1 T$ & 10, . & $I V, \ldots$ & $r .9 .0, \ldots$ & 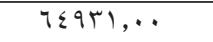 & $r \ldots$ \\
\hline$\cdot, \varepsilon)$ & $\cdot, 1 \pi$ & $10, \ldots$ & $1 v, \ldots$ & $r .9,0, \ldots$ & $7 \leq q 41, \ldots$ & $r \ldots 1$ \\
\hline$\varepsilon, .9$ & $1, \cdot 1$ & $0 \wedge \cdot, \ldots$ & $I V, \ldots$ & $1 \leqslant 179, \ldots$ & orskt,.. & $r \ldots r$ \\
\hline$\cdot, 7$. &., 10 & $V V, \ldots$ & $1 v, \ldots$ & $1 \% \wedge . q, \ldots$ & orstr,... & $r \ldots r$ \\
\hline $1, \varepsilon r$ & . Tr & $109, \ldots$ & $1 v, \ldots$ & $\| K r \mid, \ldots$ & $\sum q \backslash \wedge \wedge, \ldots$ & $r \ldots \varepsilon$ \\
\hline$\cdot, \mathrm{V} \leqslant$ & $\cdot, r_{1}$ & $1 \cdot \varepsilon, \ldots$ & $1 v, \ldots$ & 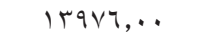 & $\leqslant 9 \wedge 0 \leqslant, \ldots$ & $r \ldots o$ \\
\hline$\cdot, \vee_{0}$ & .,YT & $110, \ldots$ & $I v, \ldots$ & 1049r,... & $01 . r \pi, \ldots$ & $r \ldots r$ \\
\hline ( & דיז, ( ) & $197, \ldots$ & $1 v, \ldots$ & $1 \leq v r q, \ldots$ & $00 . r V, \ldots$ & $r \ldots v$ \\
\hline $1, \wedge \vee$ & ., rq & rrq,... & $1 v, \ldots$ & ITRVT,.. & $010,9, \ldots$ & $r \ldots \lambda$ \\
\hline $1, \varepsilon$. & $\cdot, 71$ & $174, \ldots$ & $1 v, \ldots$ & $1107 \pi, \ldots$ & $700 \leqslant \Lambda, \ldots$ & $r \ldots q$ \\
\hline r, rt & $\cdot, r \wedge$ & $r \tau \varepsilon, \ldots$ & $1 v, \ldots$ & $\| \wedge \wedge 1, \ldots$ & $\varepsilon$ १ч,$\ldots$ & $r \cdot 1 \cdot$ \\
\hline 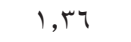 & $\cdot, \wedge)$ & $17 \varepsilon, \ldots$ & $1 v, \ldots$ & $1, .01, \ldots$ & $\varepsilon r \leqslant V \leqslant, \ldots$ & $r+11$ \\
\hline$\varepsilon, \varepsilon$. & $\cdot, 71$ & $r \cdot \wedge, \ldots$ & $1 v, \ldots$ & $v \ldots r, \ldots$ & $\Gamma \wedge .0 \Lambda, \ldots$ & $r .1 r$ \\
\hline$r, 1 T$ & $\cdot, \varepsilon$ & $19, \ldots$ & $1 v, \ldots$ & $7 . \vee 9, \ldots$ & $M 1 \leqslant 0, \ldots$ & $r \cdot 1 r$ \\
\hline 1,91 & $\cdot, \varepsilon$. & $101, \ldots$ & $1 v, \ldots$ & VTr,$\ldots$ & TVMTO,.. & $r \cdot 1 \leqslant$ \\
\hline $1, v \cdot$ & • ( & וNז,.. & $1 \mathrm{~V}, \ldots$ & $1, v 7 V, \ldots$ & OIKrT,... & $r \cdot 10$ \\
\hline $1, r$. & $\cdot, r \leq$ & $11 \mathrm{v}, \ldots$ & $1 \mathrm{~V}, \ldots$ & $q \vee V \cdot, \ldots$ & $\varepsilon \wedge r q 9, \cdots$ & $r .17$ \\
\hline$\cdot, \leqslant \wedge$ & $\cdot, 19$ & $M \cdot, \ldots$ & $1 v, \ldots$ & $r \leq q \mid \wedge, \ldots$ & ฯ人า , . & $r . I V$ \\
\hline$\cdot, \wedge \varepsilon$ & $\cdot, r)$ & $17 \pi, \ldots$ & $i v, \ldots$ & $19 \leqslant r \wedge, \cdots$ & $01970, \ldots$ & $r .11$ \\
\hline 1,7 & .,$\varepsilon \Gamma$ & $|\wedge|, \lambda$ & $1 v, \ldots$ & $1,001,01$ & 0110 r,or & المتوسط \\
\hline
\end{tabular}

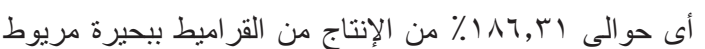

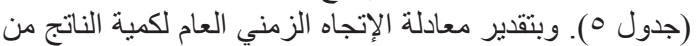

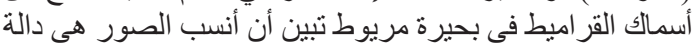

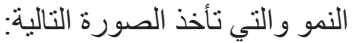

$\ln \hat{Y} i=7.47+0.037 \mathrm{Ti}$

$(88.89)^{* *}$

$(4.66)^{* *}$

$\mathrm{R}^{2}=0.561 \quad \mathrm{~F}=21.74 * *$

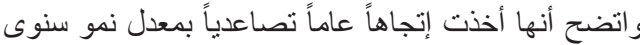

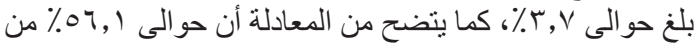

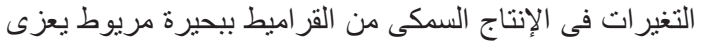

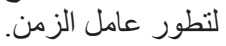

ج-مبروك الحشائش

بإستعر اض تطور الإنتاج السمكى من مبروك الحشائش

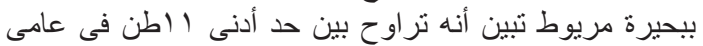

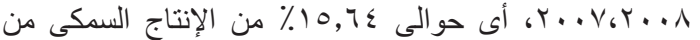

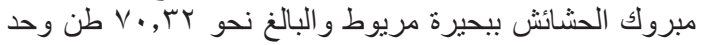

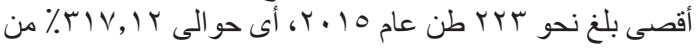

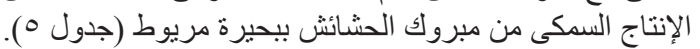

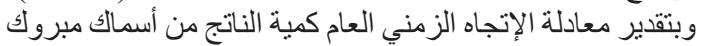
الحشائش في بحيرة مريوط تبين عدم معنوية هذا الزئن المتغير.

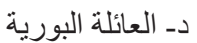
بإستعر اض تطور الإنتاج السمكى من العائلة البورية ببحيرة

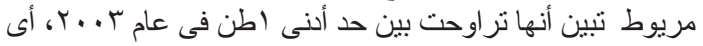

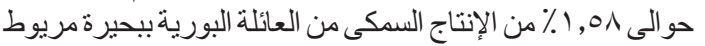

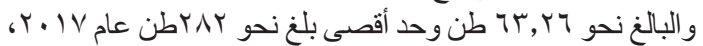

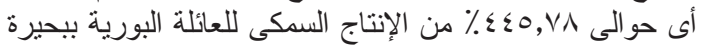

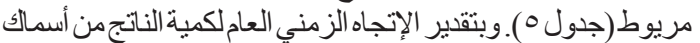
العائلة البورية في بحيرة مريوطنيف لونين عدم معنوية هذا المتغير.

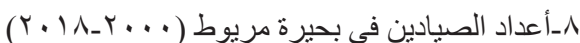

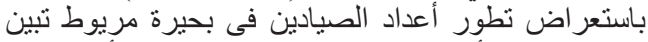

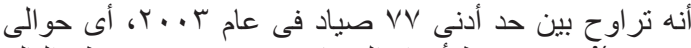

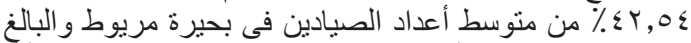

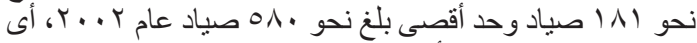

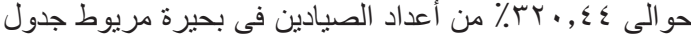

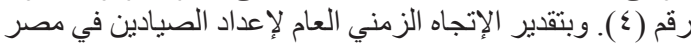
تبين عدم معنوية هذا المتغير.

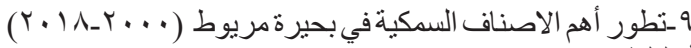
أـالبلطي

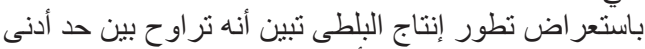

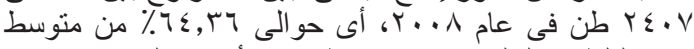

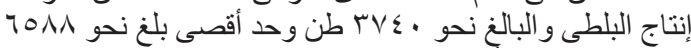

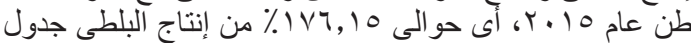

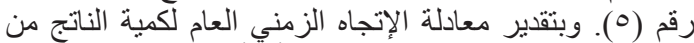

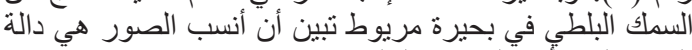
النمو و التي نأخذ الصورة البحرة التالية:

$\ln \hat{Y} \mathrm{i}=7.88+0.031 \mathrm{Ti}$

$(86.01)^{* *} \quad(3.82)^{* *}$

$\mathrm{R}^{2}=0.462 \quad \mathrm{~F}=14.59 * *$

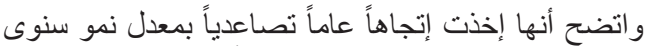

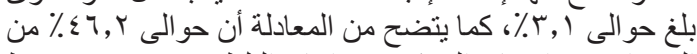

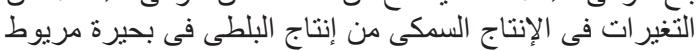
يعزى لتطور عامل الزمن.

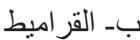

باستعراض تطور الإنتاج من القراميط نبين أنه تراوح

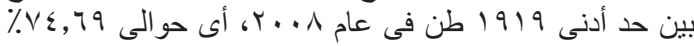

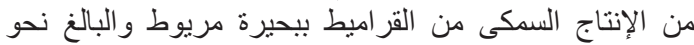

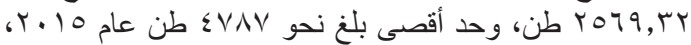

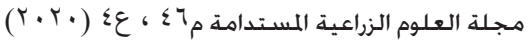




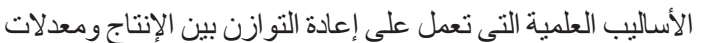

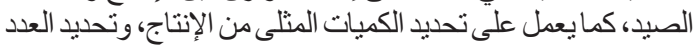

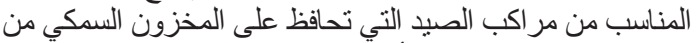

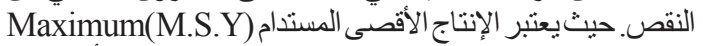
Sustainable Yield

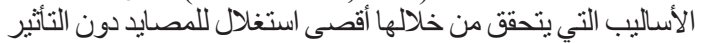

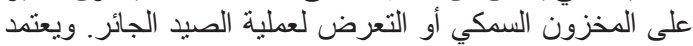

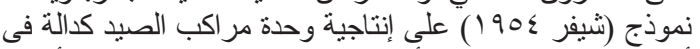

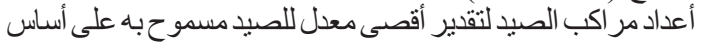

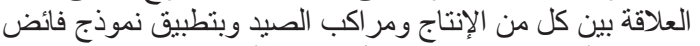

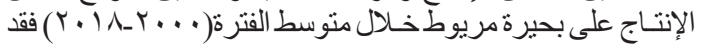

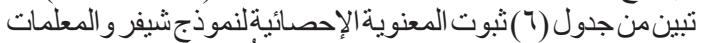

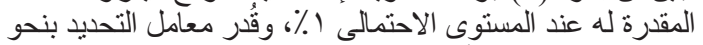

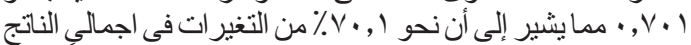

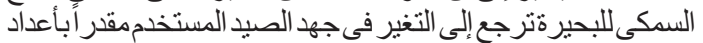

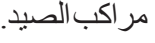

وفد تبين أن أقصى إنتاج مستدام (الغلة القصوى المستدامة)

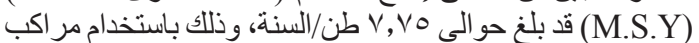

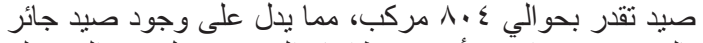

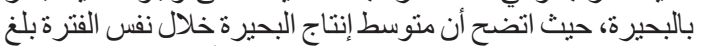

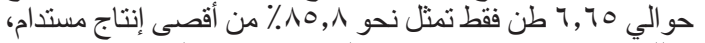

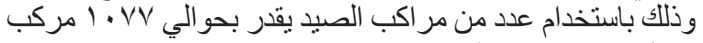

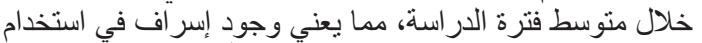

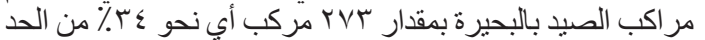
الأقصى للمر اكب في البحيرة - (جدول بل ج).

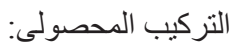

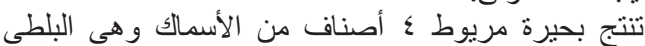

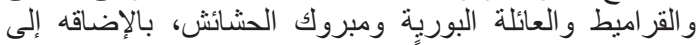

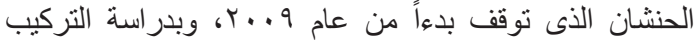

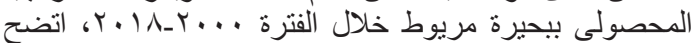

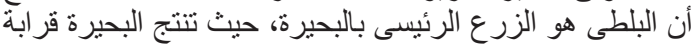

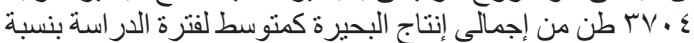

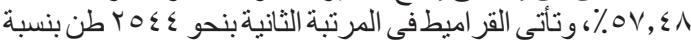

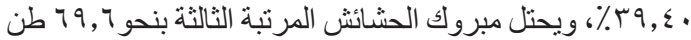

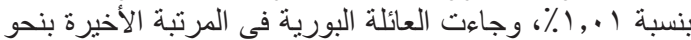

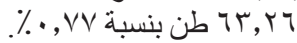

وتشير نتائج جدول (0) إلى تغير التركيب المحصولى بالبحيرة

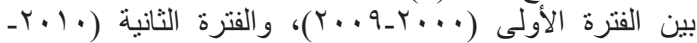

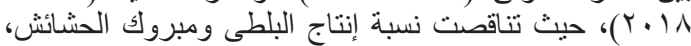

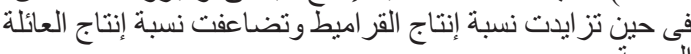
البورية.

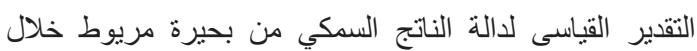

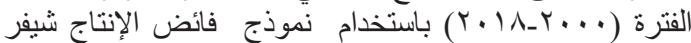
:(Schaefer) يعتبر الصيد الجائر من أهم المشاكل التي تواجه بحيرة مريوط

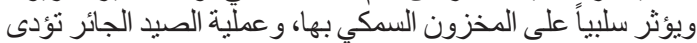

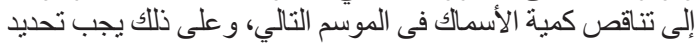

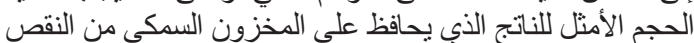

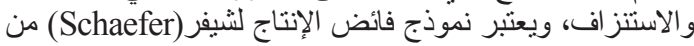

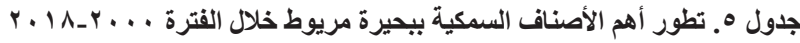

\begin{tabular}{|c|c|c|c|c|c|c|c|c|c|}
\hline$\%$ & البورية & $\%$ & الحشبائش & $\%$ & القراميط & $\%$ & البلطى & الإنتاجلى & السنة \\
\hline T,IT & Iro,.. & $\cdot, r V$ & $i v, \ldots$ & $r v, \ldots$ & זr.,.,. & Or,rᄉ & rrsו, . & 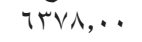 & r... \\
\hline$\cdot, 10$ & $9, \ldots$ & $\cdot, \wedge \varepsilon$ & or,.. & 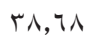 & rrqА, , & 09,9 . & rVI $\varepsilon, \ldots$ & Tr.., & $r \ldots l$ \\
\hline (ז, & Ir,.. & $1, r_{1}$ & $T \varepsilon, \ldots$ & r, & $190 \varepsilon, \ldots$ & ו & rroo,.. & or.r... & $r \ldots r$ \\
\hline$\cdot, \ldots$ & $\cdot, \ldots$ & $1,0 \varepsilon$ & $v_{0}, \ldots$ & ए৭,7т & $19 r \wedge, \ldots$ & $0 \wedge, 70$ & $r(01, \ldots$ & $\varepsilon \wedge \neg 1, \ldots$ & $r \ldots r$ \\
\hline$\cdot, \varepsilon \varepsilon$ & $r, \ldots$ & 1,99 & $1 \ldots, \cdots$ & 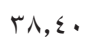 & ו ו & $0 \wedge, 9 \varepsilon$ & rqт४,.. & $0 . r \leqslant, \ldots$ & $r \ldots \varepsilon$ \\
\hline$\cdot, Y_{T}$ & $1 \leq, \ldots$ & $1, \vee \wedge$ & $9 \varepsilon, \ldots$ & rq,ov & $r \cdot q \leq, \cdots$ & $0 \wedge, 17$ & $r \cdot \vee \wedge, \cdots$ & orqr,.. & r... \\
\hline , ro & $1 \pi, \ldots$ & r.10 & $11 r, \ldots$ & $\varepsilon \cdot, \vee\urcorner$ & $r \mid r \varepsilon, \ldots$ & or,or & $r q \leq 7, \ldots$ & orl1,.. & $r \ldots r$ \\
\hline$\cdot, \cdot r$ & $1, \ldots$ & , ro & $11, \ldots$ & $\leqslant \mu, 00$ & Igrr,.. & 00,9 . & $r \leqslant T V, \ldots$ & $\varepsilon \leqslant 1 \Gamma, \ldots$ & $r \ldots v$ \\
\hline$\cdot, \cdot V$ & $r, \ldots$ & , ro & $11, \ldots$ & $\varepsilon \varepsilon, .9$ & $1919, \ldots$ & $00, \pi$ & $r \varepsilon \cdot V, \ldots$ & $\varepsilon r \circ r, \ldots$ & $r \cdots \wedge$ \\
\hline$\cdot, r)$ & $1 v, \ldots$ & $\cdot, r q$ & $17, \ldots$ & rq, r. & r)тr,.. & $T \cdot, r$. & rrtr,.. & $0011, \ldots$ & $r \ldots q$ \\
\hline$\cdot$, ז & YT,T. & $1, .7$ & $00, r$. & rq, v^ & $r \cdot \vee 9,0$. & OV, V $\varepsilon$ & $r \cdot r \leqslant, \Lambda$. & oror, r. & متوسط الفترة الأولى \\
\hline$\cdot, r V$ & $17, \ldots$ & $\cdot, r \cdot$ & $1 \wedge, \cdots$ & rq,r & rrrı,. & $7 \cdot, \cdot 9$ & roov,.. & $0919, \ldots$ & $r .1$. \\
\hline זr, & $1 \wedge, \ldots$ & $\cdot, r \wedge$ & $10, \ldots$ & 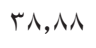 & $r) \cdot, \ldots$ & $7 ., 01$ & MYA & $0 \leqslant r v, \ldots$ & $r .11$ \\
\hline$\cdot, r \varepsilon$ & $r_{0}, \ldots$ & $\cdot, r V$ & $r \cdot, \ldots$ & $r q, .0$ & rq..,... & ס ז., & $\varepsilon \varepsilon \wedge r, \ldots$ & $V \leqslant r v, \ldots$ & $r \cdot I r$ \\
\hline$\cdot, r$. & $r r, \ldots$ & $\cdot, r \wedge$ & $r, \ldots$ & $r q, Y \leq$ & rq97, . & $7 ., 19$ & $\leqslant 097, \ldots$ & טדז,.. & $r .1 T$ \\
\hline זTr, & ro,.. & $\cdot, r \wedge$ & $r, \ldots$ & $r q, 1 \leq$ & rqr।,.. & $T \cdot, r \leqslant$ & $\varepsilon \leqslant 97, \cdots$ & $v \leq \tau r, \ldots$ & $r \cdot 1 \leqslant$ \\
\hline$r, \cdot r$ & $r \leqslant \wedge, \cdots$ & $1, \wedge 1$ & rrT,.. & r৯, q & $\varepsilon \vee \wedge \vee, \ldots$ & Or,OT & $70 \wedge 1, \ldots$ & $\mid r r \cdot 1, \ldots$ & $r .10$ \\
\hline r,IT & INr,.. & I,Ar & $107, \ldots$ & rq,or & . . & or,NI & sor,$\ldots$ & $1071, \ldots$ & $r .17$ \\
\hline$r, . q$ & rNr,.. & $1, v \varepsilon$ & $109, \ldots$ & $r \Lambda, \cdot v$ & $r \leqslant V r, \ldots$ & or, rq & $\leqslant \wedge 79, \ldots$ & $q \mid r \cdot, \ldots$ & $r . I V$ \\
\hline 1,90 & $10 \mathrm{y}, \ldots$ & $1, \wedge \vee$ & $101, \ldots$ & 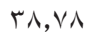 & riro,.. & or, t. & $\varepsilon r 19, \ldots$ & $\wedge .01, \ldots$ & $r .11$ \\
\hline $1, r$. & $1 \cdot \wedge, \leqslant \leq$ & $\cdot 97$ & $\wedge v, 11$ & $r_{\Lambda}, 99$ & Tו1T,07 & ov, 19 & EOrT,OT & $\vee q q ., r r$ & متوسط الفترة الثنانسة \\
\hline$\cdot, \mathrm{V} V$ & דצ, & $1, .1$ & 79,07 & rq, $\varepsilon$. & ros $\varepsilon, \wedge r$ & $O V, \Sigma \Lambda$ & $r v \cdot \varepsilon, v \varepsilon$ & $7 \leqslant \wedge \uparrow, 01$ & المتوسط \\
\hline
\end{tabular}

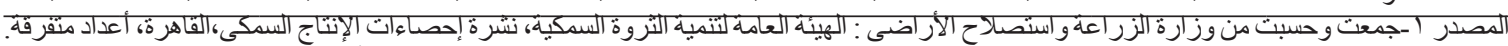
rـ الجهاز المركزى للتعبئة العامة و الإحصاء،الكتاب الإحصائى السنوى، احصاءعات الثروة السمكية فى مصر ، القاهرة، أعداد متفرقة.

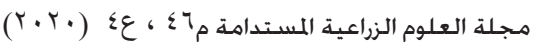




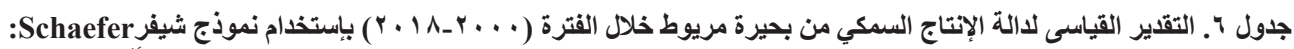

\begin{tabular}{|c|c|c|c|c|c|c|}
\hline $\mathbf{F}$ & $\mathbf{R}^{r}$ & 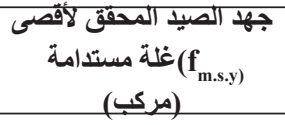 & $\begin{array}{c}\text { الإنتاج الأقصى المستدام } \\
\text { (طSY)(MSY) }\end{array}$ & الميل & الجزء المقظوع من المحور & شيفرذج \\
\hline T, , • ¿** & $\cdot, \vee \cdot 1$ & $\Lambda \cdot \varepsilon$ & v, vo & $-\cdot, \cdot 1 r$ & 19,19 & بريوط \\
\hline
\end{tabular}

الكصدر : جمعت وحسبت من نتائج تحليل البيانات الو اردة فى جدول رقم (Vchaefer بإنتخدام نموذج

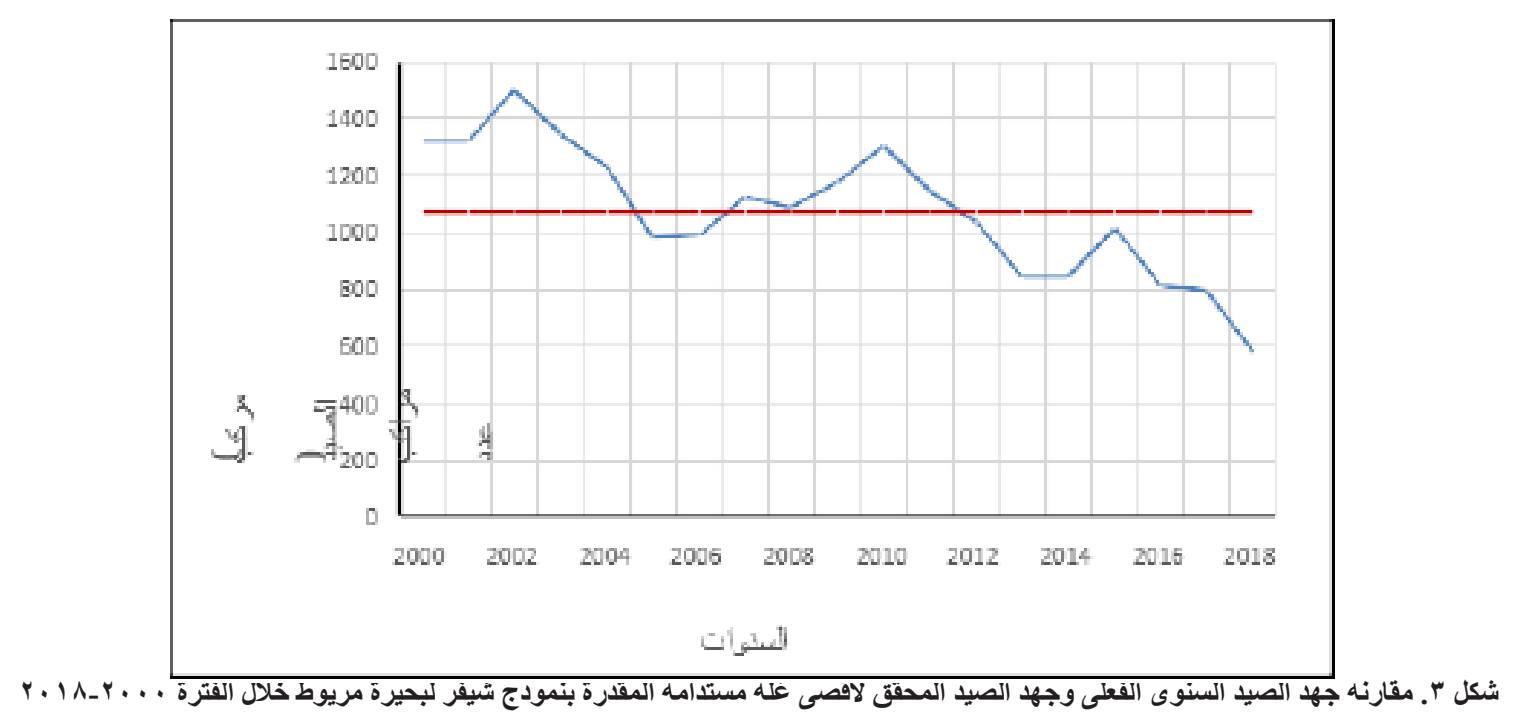

( المصدر :بيانات جدول (v)

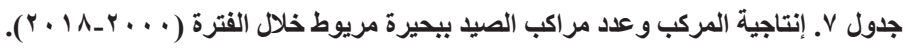

\begin{tabular}{|c|c|c|}
\hline عدد مراكب الصيد (مركب) & متوسط إنتاجية المركب (كجم/مركب) & الاسنماتر \\
\hline $\mathbf{X}$ & $\mathbf{Y}$ & الستواك \\
\hline $1 \pi 1 \wedge, \ldots$ & $\varepsilon, \wedge \varepsilon$ & $r \ldots$ \\
\hline$|r| \wedge, \ldots$ & $\varepsilon, \vee$. & $r \ldots l$ \\
\hline $1 \leqslant 99, \ldots$ & $r, 0 \leqslant$ & $r \ldots r$ \\
\hline $1 \pi \leq r, \ldots$ & retr & $r \ldots r$ \\
\hline וזr,.. & $\varepsilon, \cdot \wedge$ & $r \ldots \varepsilon$ \\
\hline $9 \wedge \vee, \ldots$ & 0,4 & r..o \\
\hline $99 \cdot, \ldots$ & $0, Y_{T}$ & $r \ldots r$ \\
\hline $11 \mathrm{r}, \ldots$ & r,q1 & $r \ldots v$ \\
\hline $1 . \wedge 7, \ldots$ & $\varepsilon, .1$ & $r \ldots \wedge$ \\
\hline $1111, \ldots$ & $\varepsilon, T \vee$ & $r \ldots q$ \\
\hline$\uparrow \cdot \wedge, \cdots$ & $\varepsilon, 0$ r & $r \cdot 1 \cdot$ \\
\hline $11 \leq 0, \ldots$ & $\varepsilon, \vee \varepsilon$ & $r \cdot 11$ \\
\hline l.r^,.. & V,17 & r. Ir \\
\hline$\wedge \leqslant 0, \ldots$ & $9, \cdot \leqslant$ & t.itr \\
\hline$\wedge \leqslant r, \ldots$ & $\wedge, \wedge \circ$ & $r \cdot 1 \varepsilon$ \\
\hline $1.1 r, \ldots$ & $\mid r, 1 \leq$ & $r \cdot 10$ \\
\hline $110, \ldots$ & $1 \cdot, 0$ & $r .17$ \\
\hline$v 90, \ldots$ & $11, \leqslant V$ & r.IV \\
\hline $0 \wedge 1, \ldots$ & IT,AV & $r \cdot 11$ \\
\hline $1 \cdot V V$ & 7,70 & المتوسط \\
\hline
\end{tabular}


لسد هذا العجز بطريقةتوفر في تكاليف التخزينو النقل وتحدث التو ازن

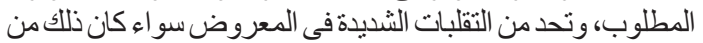

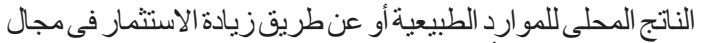

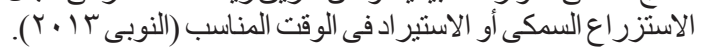

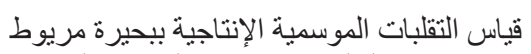

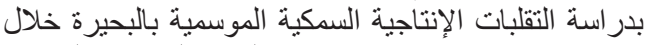

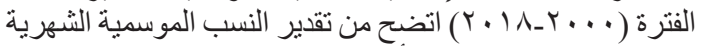

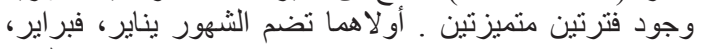

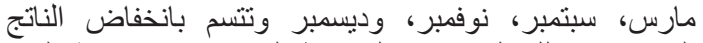

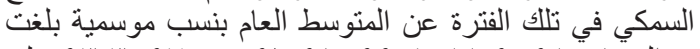

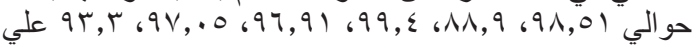
الترتيب. وقد يرجع ذللك الانخفاض عاض للناتج في ذلك الفته الفترة إلى البيات الشتوى لبعض الأسماك في بعض شهور الخفر الَّنة.

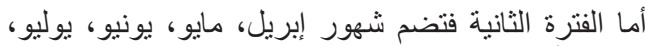

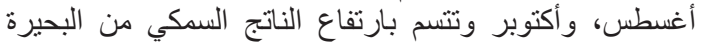

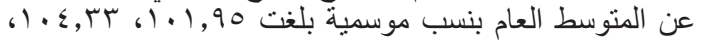

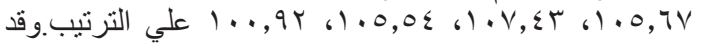

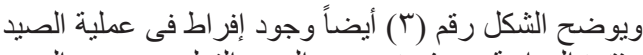

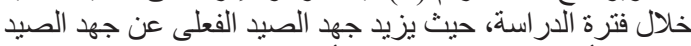

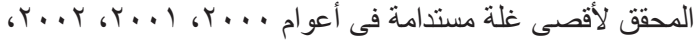

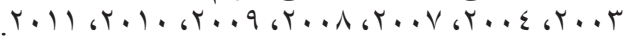
قياس التقلبات الموسمية الإنتاجية

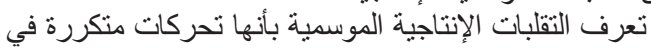

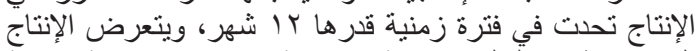

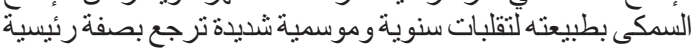

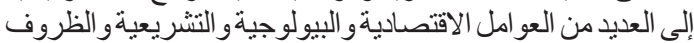

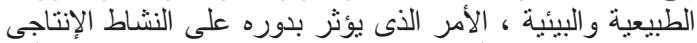

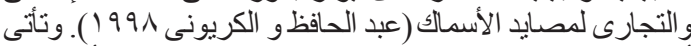

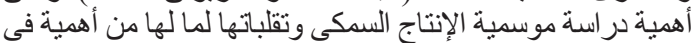

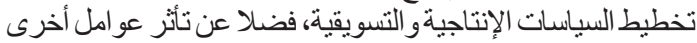

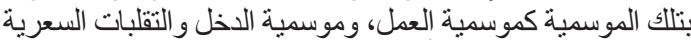

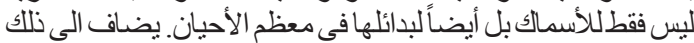

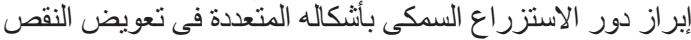

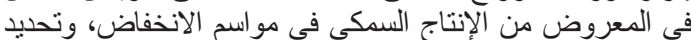

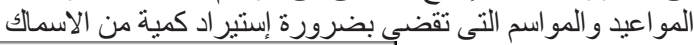

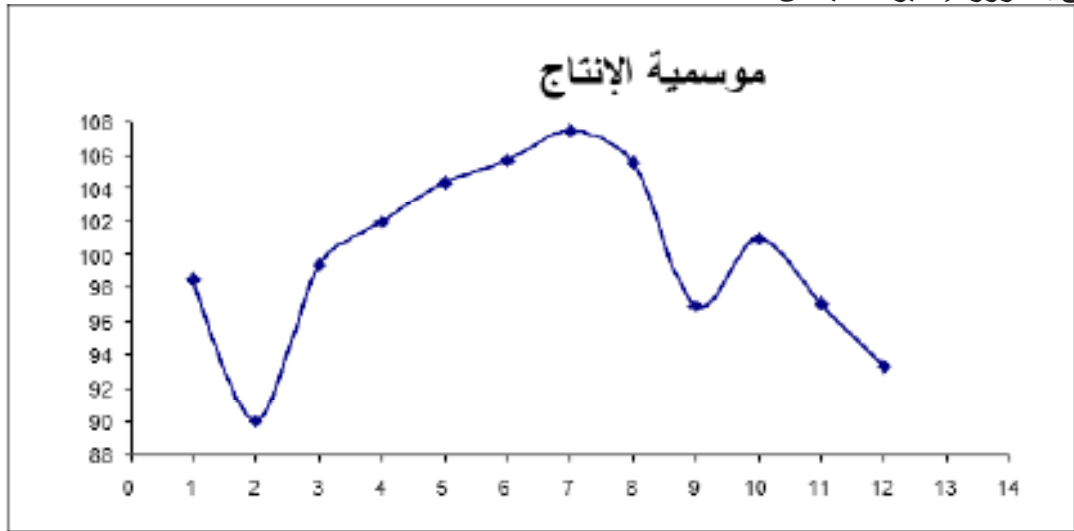

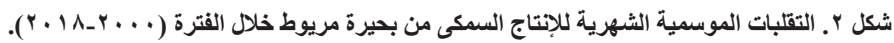

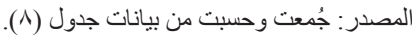

\begin{tabular}{|c|c|c|c|c|c|c|c|c|c|c|c|c|c|c|}
\hline \multicolumn{15}{|c|}{ 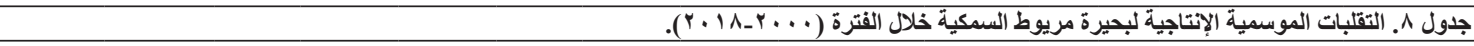 } \\
\hline المتوسط & الإجمالى & ديسمبر & نوفمبر & اكتوير & سبتمبر & أغسطس & يوليو & يونيو & مايو & ابريل & مارس & فبراير & يناير & السنة و الثهر \\
\hline orv,iv & TrAT & orv, .. & $011, \ldots$ & $007,$. & $0 \leqslant 1, \cdots$ & $0 \uparrow \wedge, .$. & or.,... & OดY & $\leqslant q \vee$ & $0.9, \cdots$ & or. & ors & 007 & $r \ldots$ \\
\hline $017, \wedge r$ & $T+$. & $0 \leqslant 7, \ldots$ & $0.0, \ldots$ & $0.0, \ldots$ & $\leq १ \leqslant, \ldots$ & $\varepsilon \vee\urcorner, .$. & $010, \ldots$ & $\varepsilon 9 \vee$ & TKV & orv, .. & sVr & $\varepsilon \cdot r$ & 010 & r... \\
\hline$\leq \leqslant 1,9 r$ & or.tr & $\varepsilon \vee q, \cdots$ & $\varepsilon \cdot r,$. & rar,,. & $\varepsilon \cdot r, \ldots$ & $\varepsilon \ldots,$. & $r 00, \ldots$ & sov & $\leq 90$ & sor,,. & $\leq 79$ & ENr & $01 \mathrm{~V}$ & $r \ldots r$ \\
\hline$\varepsilon \cdot 0, \cdot \wedge$ & $\{\wedge T\}$ & ro.,... & $r_{100}, .$. & $r \lambda \nu, .$. & $\varepsilon .7, \ldots$ & $\varepsilon \cdot r,$. & $\varepsilon \cdot r, .$. & $\leqslant r q$ & $\varepsilon r$. & га१,.. & $\$ 17$ & $\leqslant 1$. & ¿ov & $r \ldots r$ \\
\hline$\varepsilon|\Lambda, T\rangle$ & $0 . r \varepsilon$ & rлт,.. & $\varepsilon \cdot \wedge, \cdot$. & $\varepsilon . \uparrow,$. & $\varepsilon r, \ldots$ & $\varepsilon r v,$. & $\varepsilon 1 \leqslant, \cdots$ & $\varepsilon \leqslant r$ & $\varepsilon \varepsilon$. & $\varepsilon r q, \cdots$ & $\varepsilon \cdot r$ & rri & sov & $r_{\ldots} . . \varepsilon$ \\
\hline$\leqslant \leqslant 1,$. & orar & $\leqslant 17,$. & $\varepsilon r_{0}, .$. & $\varepsilon r v,$. & $\varepsilon 09, \ldots$ & $\varepsilon 09,$. & $\varepsilon \uparrow \wedge,$. & £07 & 纟od & $\varepsilon \leqslant \wedge,$. & \& T & $\varepsilon .7$ & $\varepsilon \vee v \varepsilon$ & r...o \\
\hline$\varepsilon r \xi, r_{0}$ & orll & rq), . & $\varepsilon r_{\bullet}, \ldots$ & $\varepsilon 1 r,$. & $\leqslant 01, \ldots$ & oro,... & $\{r,, \cdots$ & sor & sor & $\varepsilon \leqslant r, \cdots$ & $\$ 17$ & हाT & \&ाT & $r \ldots r$ \\
\hline rTV,vo & 纟纟ाT & rr.,. & rот,.. & ro $\leqslant, .$. & $r_{\lambda \varepsilon, \ldots}$ & rso,... & ror,.. & rso & ral & rvo,.. & ror & $r \leqslant v$ & $\varepsilon \cdot \wedge$ & $r \ldots r$ \\
\hline rTr,TV & Eror & $r \lambda_{\cdot}, \ldots$ & ros, .. & $r \leqslant \uparrow, .$. & $r \wedge \uparrow,$. & rлt,.. & roq,... & rAT & rAr & $r \wedge \wedge, .$. & ro. & TrV & $\varepsilon \cdot 1$ & $r \ldots \wedge$ \\
\hline$\{0 \vee, 0 \wedge$ & .0011 & $\varepsilon 1,, \ldots$ & ovr,.. & $009, .$. & $\varepsilon \pi, \ldots$ & $\varepsilon r \wedge, .$. & orr,.. & oro & rro & $\varepsilon 0 \wedge,$. & $\$ 17$ & roq & $\varepsilon .7$ & $r \ldots q$ \\
\hline$\varepsilon q r, r_{0}$ & 0919 & .,.,דזr & $\varepsilon r q, \ldots$ & $\varepsilon r r,$. & $\varepsilon r r, \cdots$ & $\pi$ & var,.. & vq. & \&v1 & sor, ,. & orr & rar & דre & r.l. \\
\hline \&or,, & $0 \leqslant Y Y$ & r.r,.. & $\varepsilon १ १, .$. & $\leqslant \leq \uparrow,$. & г৭१, , & $\varepsilon \pi r,$. & $091, \cdots$ & $0 \leqslant 7$ & ONY & $\varepsilon \cdot \wedge,$. & $r$. & TAT & rar & $r .11$ \\
\hline $710,1 \mathrm{~V}$ & $V \leqslant T V$ & $\vee \cdot १, \cdot$. & orr,,. & $10 \lambda, .$. & , ודוז & $V I r, .$. & $70 \leqslant,$. & 7.7 & $7 \leqslant \wedge$ & T.r,. & ory & $\sum 94$ & ox. & $r . l r$ \\
\hline זr, & צ' & $\pi+,$. & $\uparrow+\lambda,$. & $079, .$. & iro,... & $709,$. & רזr,.. & 700 & $\eta \lambda$. & $\eta{ }^{\prime} \wedge,$. & $T V \varepsilon$ & Tा & 000 & $r .1 T$ \\
\hline & v\&T & $T \leqslant r,$. & $\Delta \wedge,,$. & $r_{0}, .$. & $T \leqslant 1, \ldots$ & เพ৭,.. & ד & $7 \leqslant 1$ & THA & $\eta,,$. & ז. & $0 . £$ & 719 & $r .1 \leq$ \\
\hline $1 . r o, .1$ & $1 r+1$ & rro,.. & $u \cdot v, .$. & $i r \cdot r, .$. & $790, \ldots$ & $\| r \mid, \ldots$ & lirr,.. & 1.7. & 1101 & $117 \lambda, .$. & 1.77 & M. & qVY & $r .10$ \\
\hline VI & 1071 & oqv,... & $7 \wedge, \ldots$ & TqY, .. & $T \leqslant V, \ldots$ & VAr,... & $\vee_{\text {१८, }, .}$ & 791 & 790 & $79 \vee, .$. & 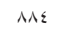 & VT. & TAT & $r .17$ \\
\hline$v_{T} \cdot, \wedge r$ & qार. & VTr,, & vrq,, & Vrז, ,. & $v v_{0}, \ldots$ & $\wedge 19, .$. & voq,... & 710 & VTu & vor,... & V10 & $\wedge 1$. & vor & r.lv \\
\hline TYוo. & 1.01 & $7 \leqslant q,$. & or.,.. & $707,$. & Vrr, , & $7.7,$. & $v_{00}, .$. & Tor & $\gamma \cdot \varepsilon$ & $v \leqslant,, \cdots$ & 790 & 010 & VTr & r.ls \\
\hline \multirow[t]{4}{*}{$0 \leq 0, \wedge \leq$} & $70 \leqslant 9$ & $0.9, Y^{4}$ & or $q, \mathrm{r} \varepsilon$ & $00, \wedge \varepsilon \varepsilon$ & OrA,90 & ovı,. o & $0 \wedge 7, r v$ & ort & 079 & $007, \leqslant V$ & $0 \leqslant r$ & $\leqslant 10$ & orv & المتوسط \\
\hline & Eror & $r_{\mu}, \ldots$ & rot,... & $r \leqslant \uparrow,$. & $r_{\lambda \varepsilon,}$, & rлт,... & roo,.. & rAT & rto & rro,. & $r$. & TAY & rar & الحد الأدنى \\
\hline & $1 K r \cdot 1$ & Vrr,, & $11 \cdot v, .$. & Ir.r,.. & $v_{90}, \ldots$ & $\| r \mid, \ldots$ & lirr,.. & 1.7 & 1101 & $117 \wedge, .$. & 1.77 & $\wedge$. & GYY & الحد الأعلى \\
\hline & & $q r, r ৭ q$ & qv,.०. & $1 \ldots, q 1 \mathrm{~V}$ & $97,9.7$ & $1.0,017$ & $1 \cdot v, \varepsilon r_{0}$ & 1.0 & $1 . \varepsilon$ & $1.1,9 \leqslant 9$ & 99 & $\Lambda \Lambda$ & 91 & النسب الموسمية \\
\hline
\end{tabular}

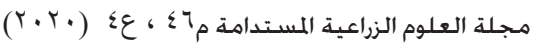


El-Gammal FI, Mehanna SF (1999). Maximum Sustainable Yield of the demersal fish resources exploited by trawling in the Gulf of Suez with special reference to Shrimp fishery. The role of Science in the Development of Egyptian Society and Environment, 23-24: 198-210.

Sparre P, Venema SC (1988). Introduction to Tropical Fish Stock. Assessment. Part 1. Fisheries and Aquaculture Department, FAO Fisheries Technical pages, No 306, Rome, FAO, P 280.
يعزى ذللك إلى أن معظم الأسماك تبدأ في التكاثر و التوالد في الصيف فتنمو ويظهر أثرها في تللك الثهور. (جدول م) وشكل

التوصبات

$$
\begin{aligned}
& \text { إتساقاً مع ماتقدم من نتائج بحثية، فيمكن التوصية بما يلى: }
\end{aligned}
$$

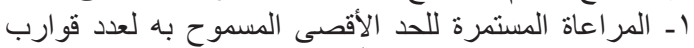

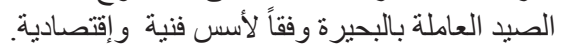

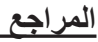

الجهاز المركزى للتعبئة العامة و الإحصاء، النشرة السنوية لإحصائيات الإنتاج السمكى، أعداد متفرقة.

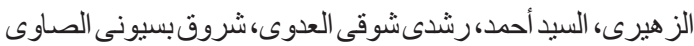

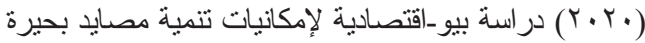

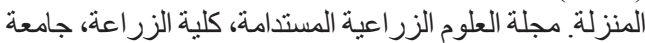

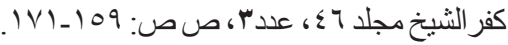

الزهيرى، السيد أحمد، رشدى شوقى العدوى و شروق بسيونى

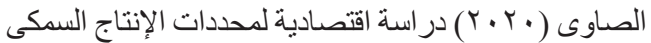
لبحيرة البرلس. مجلة العلوم الزراعة دراعية المستدامة، كلية الإنية

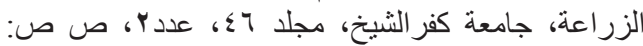
. $1 \leqslant \tau_{-1} 1$ \%

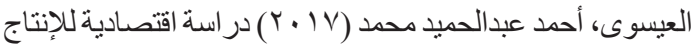

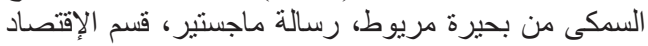
و إدارة الأعمال الزر اعية، كلية الزر اعة جامعة الإسكندرية.

مزروع، ياسر سيد أحمد و رشدى شوقى العدوى (10 • ب) در اسة

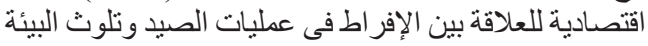

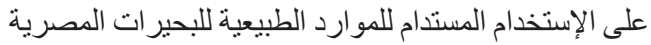

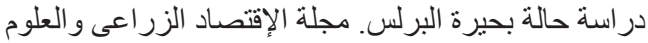

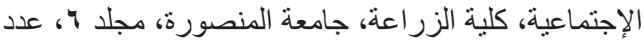
r Y T

منظمة الأغذية والزر اعة العالمية (ع . . ץ) حالة الموارد السمكية

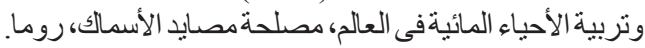

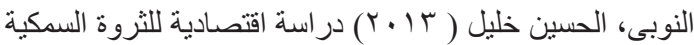

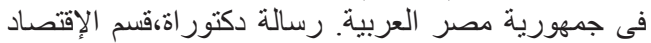
و إدارة الأعمال الزر اعية، كلية الزر اعة، جامعة الإسكندرية. 


\section{An Economic Study of The Impact of Overexploitation on Sustainable Use for Fish Resources in the Egyptian Lake Mariout}

\section{Roshdy Shawky Eladawy}

Department of Agricultural Economics- Faculty of Agriculture - Kafr El- Sheikh

University

$\mathbf{O}$ VEREXPLOITATION is one of the most important problems facing Lake Mariout, due to its negative impact on the quantity of fish production in the coming seasons, and the lake also faces the causes that led to a decrease in its area from 204 thousand feddans in 1798 to 17 thousand feddans in 2018. The research problem is represented in the fluctuation in the total fish production of the lake and the fluctuation of the main culture production (tilapia) from it to the total fish production in them from $61.38 \%$ as a maximum in 2001 to $52.38 \%$ as a minimum in 2000 . The research aims mainly to identify the effect of overfishing on the sustainable use of fishery resources in the Egyptian Lake Mariout. Surplus production models It was found from the estimated results that there is wasteful use of fishing boats in the lake by 273 boats, or about $34 \%$ of the maximum number of boats in the lake, as the actual fishing effort exceeded the fishing effort achieved for the maximum sustainable yield, which indicates. The study recommends the continuous review of the maximum permissible number of fishing boats operating in the lake according to technical and economic principles, in order to ensure sustainable use of the materials in the lake, while studying the allocation of fishing boats according to the fishing effort and including them with permits for fishing, in cooperation between the lake administration, fish cooperatives and elders of fishermen.

Keywords: Overexploitation, seasonal fluctuations, Schaefer model, fish production, maximum yield effort, Lake Mariout, Fish resources. 